\title{
EQUILIBRIUM OF TWO RODS IN CONTACT UNDER PRESSURE
}

\author{
by S. TURZI \\ (Dipartimento di Matematica, Politecnico di Milano, Piazza Leonardo da Vinci 32, 20133 \\ Milano, Italy) \\ M. ZOPPELLO and D. AMBROSI ${ }^{\mathbb{D} \dagger}$ \\ (DISMA, Politecnico di Torino, Corso Duca Degli Abruzzi 24, 10129 Torino, Italy)
}

[Received 24 February 2020. Revised 10 November 2020. Accepted 10 November 2020]

\begin{abstract}
Summary
We study the equilibrium of a mechanical system composed by two rods that bend under the action of a pressure difference; they have one fixed endpoint and are partially in contact. This system can be viewed as a bi-valve made by two smooth leaflets that lean on each other. We obtain the balance equations of the mechanical system exploiting the principle of virtual work and the contact point is identified by a jump condition. The problem can be simplified exploiting a first integral. In the case of quadratic energy, another first integral exists: its peculiarity is discussed and a further reduction of the equations is carried out. Numerical integration of the differential system shows how the shape of the beams and the position of the contact point depend on the applied pressure. For small pressure, an asymptotic expansion in a small parameter allows us to find an approximate solutions of polynomial form which is in surprisingly good agreement with the solution of the original system of equations, even beyond the expected range of validity. Finally, the asymptotics predicts a value of the pressure that separates the contact from the no-contact regime of the beams that compares very well with the one numerically evaluated.
\end{abstract}

\section{Introduction}

Various theories of elastic rods, with different level of sophistication, are used to describe the deformation of three-dimensional (3D) elastic slender bodies, with one-dimension (1D) much larger than the other two. These models are usually considered a first step towards the understanding of a number of physical and bio-mechanical phenomena. Despite their apparent simplicity, contact problems among elastic rods are still challenging due to the unilateral constraints and the free boundary conditions (the contact region is a priori unknown) they introduce.

In the framework of interacting elastic bodies, the problem of determining equilibrium states of inextensible strings in contact, provides a rich paradigm. In particular the problem of finding these states when the applied load is only a normal pressure of constant intensity is a fascinating example. In this case, every beam is called a velaria ('like sail in the wind') (1). The adhesion problem of a rod with a rigid surface, or confined by a rigid external container, has been extensively studied (2-7); on the other hand, the problem of two elastic rods, confined by their mutual interaction, has received less attention $(\mathbf{8}, \mathbf{9})$. In both cases, adhesion boundary conditions arise from a variational formulation, and we will follow this approach too. Similar problems have been numerically addressed, for a single buckled elastica (10), as a function of the compressive

$\dagger<$ davide.ambrosi@polito.it $>$

Q. Jl Mech. Appl. Math, Vol. 73. No. 4 (C) The Author, 2021. Published by Oxford University Press; all rights reserved. For Permissions, please email: journals.permissions@oup.com. Advance Access publication 4 January 2021. doi:10.1093/q.jmam/hbaa016 
exerted force, and analytically in the case of two nested elastic rings (8). A remarkable example is provided by the sophisticated rod theories that have been used to investigate DNA conformations $(11,12)$, where the DNA molecule is modelled as a structure made of two interacting elastic strands. Similar models are used for closely related problems such as delamination from elastic substrates (13) and peeling (3), where the contact is enforced through an adhesive potential rather than a geometric constraint. The propagation of an arterial dissection is an important biological application in this context and it has been extensively studied via numerical simulations $(14,15)$.

Here, we study a planar system composed of two inextensible elastic beams, hinged at one end, which are subject to a pressure difference pushing the rods upwards, so that equilibrium is possible when they are in contact for a portion of their length. We assume there is no friction between the rods. The mechanical system can be understood as a 2D cartoon of two parachutes in contact (without wires) or a mitral valve without chordae tendineae.

The numerical literature of rods in contact is ample, also in a biological context. Most of the studies consider 3D systems, finite cross-sections and include friction/adhesion forces in the contact region (16-18). Due to their intrinsic complexity, the numerical solution of the resulting equations is the only option available in such cases. By contrast, our aim is to formulate a minimalistic 1D model able to capture some key physical features and simple enough to be analytically tractable. Despite its simplicity, a 1D model gives a valuable insight into the mechanics of the system. Non-trivial questions that can be answered by this simple model are, for example, the determination of the threshold pressure that the attached beams can sustain. In the same vein, what is the length of the free portion as a function of the pressure and of other material parameters?

We ideally split each rod in its attached and unattached portion and the equilibrium equations are obtained exploiting the principle of virtual work, written for rods under the action of an external constant pressure (with dimensions of force per unit length). After taking the first variation of the energy functional, we obtain a system of 24 first-order ordinary differential equations, supplemented by suitable boundary conditions and two nonlinear algebraic equations that define the curvilinear coordinates of the attachment point. We show that balance equations have a first integral, that we exploit to simplify the differential system. When quadratic energy is assumed, there is one more first integral. The peculiarity of this first integral and its relation with the contact condition (that might yield indifferent equilibrium configurations) are discussed. First integrals of the system are then exploited up to reduce further the problem to two scalar (very nonlinear) second-order equations in the angular coordinate. In section 4 , we carry out an asymptotic expansion of the solution in terms of small $\alpha$, a non-dimensional parameter that characterises the pressure regime versus the bending stiffness of the material. Thus, we obtain a polynomial representation of the solution that fits rather well with the numerical solution of the original problem. More importantly, we exploit a reduced basis expansion to find approximate solutions in the symmetric version of the problem and obtain an asymptotic value for the critical pressure load above which the rods are no longer in contact.

Numerical results are reported in section 5: at variance of pressure and bending stiffness, the rods take different shape and attach to each other in different points. The equilibrium profiles, as given by the asymptotic approximation, compares in an excellent way with the numerical fully nonlinear ones. A contact equilibrium configuration exists up to a critical pressure, beyond which the leaflets open up: the theoretical and numerical critical value are in very good agreement. 


\section{The two leaflet system}

\subsection{Kinematics}

We consider a mechanical system composed by two inextensible leaflets that are in contact for a portion of their length. For mathematical convenience, each physical leaflet is treated as two connected rods (see Fig. 1). We therefore conveniently extend the approach outlined in (8) and introduce four curves in the plane: the former ones describe the non-attached portion of the leaflets, subject to a pressure difference, while the latter accounts for the partially attached configuration,

$$
\mathbf{r}_{i}\left(s_{i}\right):\left[0, \bar{s}_{i}\right] \rightarrow \mathbb{R}^{2}, \quad i=1,2,3,4
$$

where $\mathbf{r}_{i}\left(s_{i}\right)=\left(x\left(s_{i}\right), y\left(s_{i}\right)\right)$ and in the mutual attachment point (the interface)

$$
\mathbf{r}_{1}\left(\bar{s}_{1}\right)=\mathbf{r}_{2}\left(\bar{s}_{2}\right)=\mathbf{r}_{3}\left(\bar{s}_{3}\right)=\mathbf{r}_{4}\left(\bar{s}_{4}\right)
$$

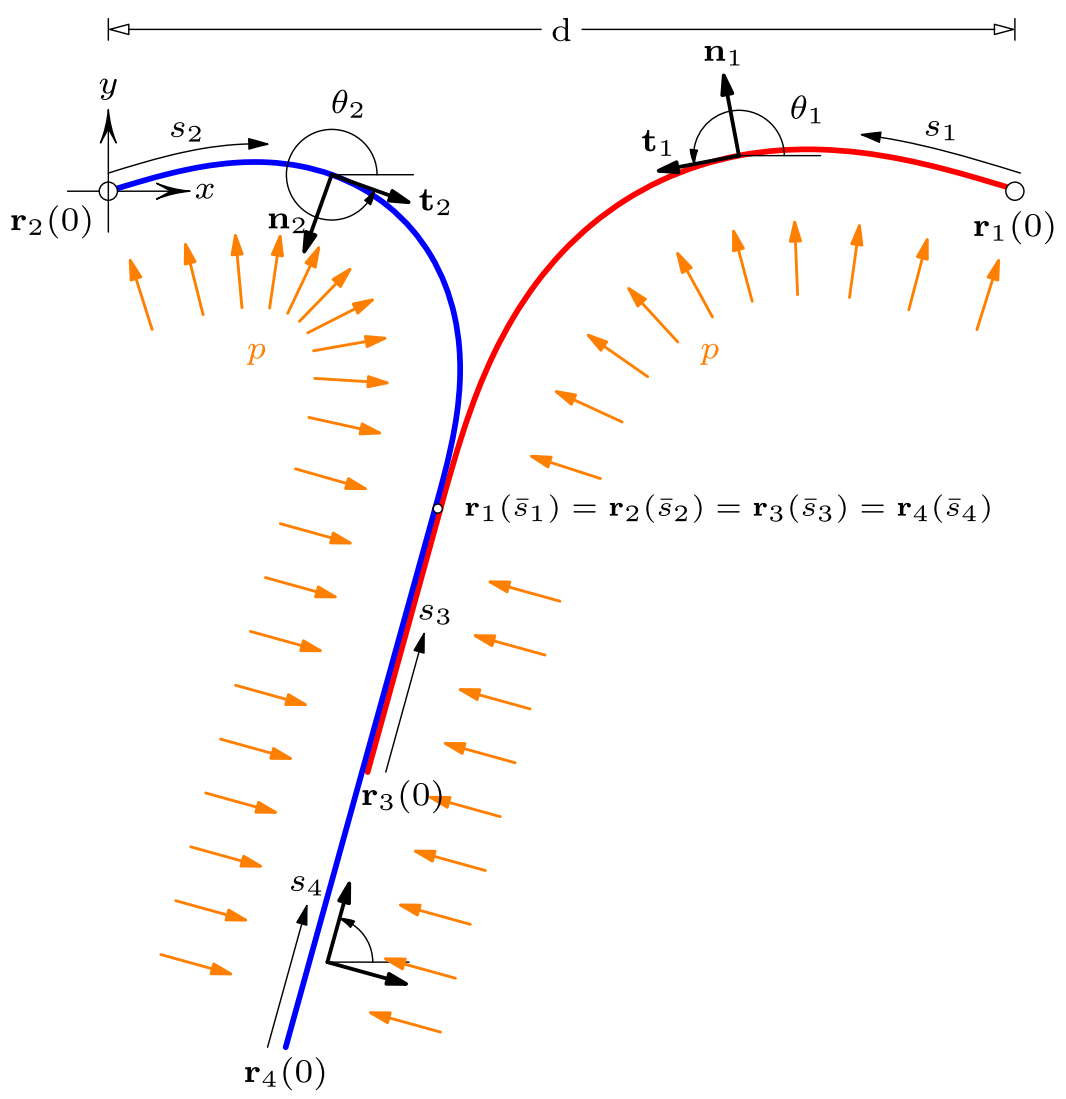

Fig. 1 The two leaflets have a fixed endpoint and partially overlap, starting from the common point $\mathbf{r}_{1}\left(\bar{s}_{1}\right)=$ $\mathbf{r}_{2}\left(\bar{s}_{2}\right)$. Orange arrows show the constant pressure acting on the lower side of the system and pushing the rods upwards. 
The rods $\mathbf{r}_{1}$ and $\mathbf{r}_{2}$ have a fixed endpoint

$$
\mathbf{r}_{1}(0)=\mathbf{r}_{1}^{0}, \quad \mathbf{r}_{2}(0)=\mathbf{r}_{2}^{0}
$$

while the endpoints $\mathbf{r}_{3}(0)$ and $\mathbf{r}_{4}(0)$ are free (see Fig. 1). At variance with the three-rod model employed in (8), our four-rod model accounts for the free sliding of one rod against the other. In so doing, we implicitly assume that no friction occurs among the rods and possible equilibrium will be provided by balance of normal force and null tangential force.

A unit tangent vector $\mathbf{t}_{i}=\left(\cos \left(\theta_{i}\right), \sin \left(\theta_{i}\right)\right)$ is uniquely defined in every point of a curve, where $\theta_{i}\left(s_{i}\right)$ is the angle between the tangent vector and the $x$-axis. Normal unit vectors are defined as $\mathbf{n}_{i}=\left(\sin \left(\theta_{i}\right),-\cos \left(\theta_{i}\right)\right)$ so that $\mathbf{t}_{i}^{\prime}=-\mathbf{n}_{i} \theta_{i}^{\prime}$. In the attachment point the tangent vector is smooth:

$$
\theta_{1}\left(\bar{s}_{1}\right)=\theta_{2}\left(\bar{s}_{2}\right)=\theta_{3}\left(\bar{s}_{3}\right)+\pi=\theta_{4}\left(\bar{s}_{4}\right)+\pi .
$$

We adopt arc-length parametrizations, so that

$$
\bar{s}_{1}+\bar{s}_{3}=\ell_{1}, \quad \bar{s}_{2}+\bar{s}_{4}=\ell_{2},
$$

where $\ell_{1}$ and $\ell_{2}$ are the rods lengths. Moreover the following relations hold:

$$
\mathbf{n}_{1}\left(\bar{s}_{1}\right)=\mathbf{n}_{2}\left(\bar{s}_{2}\right)=-\mathbf{n}_{3}\left(\bar{s}_{3}\right)=-\mathbf{n}_{4}\left(\bar{s}_{4}\right)
$$

while

$$
\mathbf{t}_{1}\left(\bar{s}_{1}\right)=\mathbf{t}_{2}\left(\bar{s}_{2}\right)=-\mathbf{t}_{3}\left(\bar{s}_{3}\right)=-\mathbf{t}_{4}\left(\bar{s}_{4}\right) .
$$

\subsection{Equilibrium equations}

The inextensible rods store elastic energy only because of bending

$$
\tilde{w}_{i}\left(\theta_{i}^{\prime}, \bar{s}_{i}\right)=\int_{0}^{\bar{s}_{i}} w_{i}\left(\theta_{i}^{\prime}\right) d s_{i}
$$

where $w_{i}\left(\theta_{i}^{\prime}\right), i=1,2,3,4$ are convex functions of the arguments, with the unique minimum in $\theta_{i}=0$. Of course, $\left(w_{1}\left(\theta_{1}^{\prime}\right), w_{3}\left(\theta_{3}^{\prime}\right)\right)$ and $\left(w_{2}\left(\theta_{2}^{\prime}\right), w_{4}\left(\theta_{4}^{\prime}\right)\right)$ are the same function except for the domain, because they represent the same physical rod.

We append the energy the inextensibility constraints, and we get

$$
W\left(\mathbf{r}_{i}, \theta_{i}^{\prime}, \mathbf{N}_{i}, \bar{s}_{i}\right)=\sum_{i=1}^{4}\left(\tilde{w}_{i}-\int_{0}^{\bar{s}_{i}} \mathbf{N}_{i} \cdot\left(\mathbf{r}_{i}^{\prime}-\mathbf{t}_{i}\right) d s_{i}\right),
$$

where $\mathbf{N}_{i}\left(s_{i}\right)=\left(N_{i}^{x}\left(s_{i}\right), N_{i}^{y}\left(s_{i}\right)\right)$ is the (unknown) reaction force of the $i$ th rod.

In our system a pressure difference applies to the rods, that is, $\mathbf{r}_{1}$ and $\mathbf{r}_{2}$. The virtual work of the external forces reads

$$
\delta L=\int_{0}^{\bar{s}_{1}} p \mathbf{n}_{1} \cdot \delta \mathbf{r}_{1} d s_{1}-\int_{0}^{\bar{s}_{2}} p \mathbf{n}_{2} \cdot \delta \mathbf{r}_{2} d s_{2} .
$$

The external forces per unit length, $p \mathbf{n}_{i}$, are not related to the reactive forces due to the rod contact: they represent the active external constant pressure difference acting perpendicularly on both rods. Furthermore, $p \mathbf{n}_{i}$ is neither conservative nor dissipative. 
In principle, if one considers separately the mechanics of the attached rods, there is a reaction force between rod 3 and rod 4 . However, in the attached portion of the system we give up representing the force balance of every single rod and, for the sake of simplicity, we only consider the overall system composed by three elements: 'rod 1', 'rod 2' and 'rod 3+4'. For the action-reaction principle, internal actions balance in every subsystem and are therefore immaterial in our representation. By contrast, the reaction force should be explicitly included if one were interested in modelling, for instance, the friction among the rods, so that the contact could no longer be considered as an ideal constraint. Therefore, the only non-vanishing contribution to the virtual work (10) comes from the free portions of the leaflets.

The principle of virtual work $\delta W=\delta L$ writes explicitly

$$
\begin{aligned}
& \sum_{i=1}^{4}\left(\int_{0}^{\bar{s}_{i}} \frac{d w_{i}}{d \theta_{i}^{\prime}} \delta \theta_{i}^{\prime} d s_{i}-\int_{0}^{\bar{s}_{i}} \delta \mathbf{N}_{i} \cdot\left(\mathbf{r}_{i}^{\prime}-\mathbf{t}_{i}\right)-\int_{0}^{\bar{s}_{i}} \mathbf{N}_{i} \cdot\left(\delta \mathbf{r}_{i}^{\prime}+\mathbf{n}_{i} \delta \theta_{i}\right)\right) \\
& \quad+\sum_{i=1}^{4}\left[w_{i}\left(\theta_{i}^{\prime}\right)-\mathbf{N}_{i} \cdot\left(\mathbf{r}_{i}^{\prime}-\mathbf{t}_{i}\right)\right]_{\bar{s}_{i}} \delta \bar{s}_{i}=\int_{0}^{\bar{s}_{1}} p \mathbf{n}_{1} \cdot \delta \mathbf{r}_{1} d s_{1}-\int_{0}^{\bar{s}_{2}} p \mathbf{n}_{2} \cdot \delta \mathbf{r}_{2} d s_{2}
\end{aligned}
$$

After integration by parts, we get

$$
\begin{aligned}
\sum_{i=1}^{4} & \left(-\int_{0}^{\bar{s}_{i}} \frac{d^{2} w_{i}}{d^{2} \theta_{i}^{\prime}} \theta_{i}^{\prime \prime} \delta \theta_{i} d s_{i}+\left[\frac{d w_{i}}{d \theta_{i}^{\prime}} \delta \theta_{i}\right]_{0}^{\bar{s}_{i}}\right. \\
& \left.-\int_{0}^{\bar{s}_{i}} \delta \mathbf{N}_{i} \cdot\left(\mathbf{r}_{i}^{\prime}-\mathbf{t}_{i}\right)+\int_{0}^{\bar{s}_{i}} \mathbf{N}_{i}^{\prime} \cdot \delta \mathbf{r}_{i}-\left[\mathbf{N}_{i} \cdot \delta \mathbf{r}_{i}\right]_{0}^{\bar{s}_{i}}-\int_{0}^{\bar{s}_{i}} \mathbf{N}_{i} \cdot \mathbf{n}_{i} \delta \theta_{i} d s_{i}\right) \\
& +\sum_{i=1}^{4}\left[w_{i}\left(\theta_{i}^{\prime}\right)-\mathbf{N}_{i} \cdot\left(\mathbf{r}_{i}^{\prime}-\mathbf{t}_{i}\right)\right]_{\bar{s}_{i}} \delta \bar{s}_{i}=\int_{0}^{\bar{s}_{1}} p \mathbf{n}_{1} \cdot \delta \mathbf{r}_{1} d s_{1}-\int_{0}^{\bar{s}_{2}} p \mathbf{n}_{2} \cdot \delta \mathbf{r}_{2} d s_{2} .
\end{aligned}
$$

The Euler-Lagrange equations of the system (12) are to be obtained by independent variations of the fields of the energy functional (9): positions, angles, reactions and the curvilinear coordinates $\bar{s}_{i}$. However, the variations $\delta \mathbf{r}_{i}\left(\bar{s}_{i}\right)$ and $\delta \theta_{i}\left(\bar{s}_{i}\right)$, appearing in (12) after integration by parts, are not independent. To decouple the increments, we observe that the total variation of $\theta_{i}$, say $\delta \bar{\theta}_{i}$, up to first order is composed by two terms (19)

$$
\delta \bar{\theta}_{i}=\left(\theta_{i}\left(\bar{s}_{i}+\delta \bar{s}_{i}\right)+\delta \theta_{i}\left(\bar{s}_{i}+\delta \bar{s}_{i}\right)\right)-\theta\left(\bar{s}_{i}\right)=\theta^{\prime}\left(\bar{s}_{i}\right) \delta \bar{s}_{i}+\delta \theta_{i}\left(\bar{s}_{i}\right),
$$

and, analogously,

$$
\delta \overline{\mathbf{r}}_{i}=\mathbf{r}^{\prime}\left(\bar{s}_{i}\right) \delta \bar{s}_{i}+\delta \mathbf{r}_{i}\left(\bar{s}_{i}\right) .
$$

Moreover, from the conditions (2) and (5) the following relations follow

$$
\delta \mathbf{r}_{1}\left(\bar{s}_{1}\right)=\delta \mathbf{r}_{2}\left(\bar{s}_{2}\right)=\delta \mathbf{r}_{3}\left(\bar{s}_{3}\right)=\delta \mathbf{r}_{4}\left(\bar{s}_{4}\right), \quad \delta \bar{s}_{3}=-\delta \bar{s}_{1}, \quad \delta \bar{s}_{4}=-\delta \bar{s}_{2}
$$


Using (13) to represent $\delta \theta_{i}\left(\bar{s}_{i}\right)$ and $\delta \mathbf{r}_{i}\left(\bar{s}_{i}\right),(12)$ rewrites

$$
\begin{aligned}
& \sum_{i=1}^{4}\left(-\int_{0}^{\bar{s}_{i}} \frac{d^{2} w_{i}}{d^{2} \theta_{i}^{\prime}} \theta_{i}^{\prime \prime} \delta \theta_{i} d s_{i}+\frac{d w_{i}}{d \theta_{i}^{\prime}}\left(\bar{s}_{i}\right) \delta \bar{\theta}_{i}-\frac{d w_{i}}{d \theta_{i}^{\prime}}(0) \delta \theta_{i}(0)\right) \\
& \quad+\sum_{i=1}^{4}\left(-\int_{0}^{\bar{s}_{i}} \delta \mathbf{N}_{i} \cdot\left(\mathbf{r}_{i}^{\prime}-\mathbf{t}_{i}\right)+\int_{0}^{\bar{s}_{i}} \mathbf{N}_{i}^{\prime} \cdot \delta \mathbf{r}_{i}-\mathbf{N}_{i}\left(\bar{s}_{i}\right) \cdot \delta \overline{\mathbf{r}}_{i}+\mathbf{N}_{i}(0) \cdot \delta \mathbf{r}_{i}(0)-\int_{0}^{\bar{s}_{i}} \mathbf{N}_{i} \cdot \mathbf{n}_{i} \delta \theta_{i} d s_{i}\right) \\
& \quad+\sum_{i=1}^{4}\left[w_{i}\left(\theta_{i}^{\prime}\right)-\theta_{i}^{\prime} \frac{d w_{i}}{d \theta_{i}^{\prime}}-\mathbf{N}_{i} \cdot\left(\mathbf{r}_{i}^{\prime}-\mathbf{t}_{i}\right)+\mathbf{N}_{i} \cdot \mathbf{r}_{i}^{\prime}\right]_{\bar{s}_{i}} \delta \bar{s}_{i} \\
& =\int_{0}^{\bar{s}_{1}} p \mathbf{n}_{1} \cdot \delta \mathbf{r}_{1} d s_{1}-\int_{0}^{\bar{s}_{2}} p \mathbf{n}_{2} \cdot \delta \mathbf{r}_{2} d s_{2} .
\end{aligned}
$$

Now all independent variations can be carried out so that the following differential equations are obtained

$$
\begin{aligned}
& \frac{d^{2} w_{i}}{d^{2} \theta_{i}^{\prime}} \theta_{i}^{\prime \prime}+\mathbf{N}_{i} \cdot \mathbf{n}_{i}=0, \quad i=1,2,3,4 \\
& \mathbf{N}_{1}^{\prime}=p \mathbf{n}_{1}, \quad \mathbf{N}_{2}^{\prime}=-p \mathbf{n}_{2}, \quad \mathbf{N}_{3}^{\prime}=0, \quad \mathbf{N}_{4}^{\prime}=0 \\
& \mathbf{r}_{i}^{\prime}=\mathbf{t}_{i}, \quad i=1,2,3,4 .
\end{aligned}
$$

which coincide with the classical equations of rod theory $(\mathbf{1}, \mathbf{2 0})$. On the basis of the assumptions (2) and (3), the system of equations is supplemented by the boundary conditions

$$
\begin{array}{r}
\mathbf{r}_{1}(0)=\mathbf{r}_{1}^{0}, \quad \mathbf{r}_{2}(0)=\mathbf{r}_{2}^{0}, \quad \mathbf{N}_{3}(0)=0, \quad \mathbf{N}_{4}(0)=0, \\
\frac{d w_{i}}{d \theta_{i}}(0)=0, \quad i=1,2,3,4 .
\end{array}
$$

Note that, because of the assumptions on the bending energy function, the last condition is equivalent to $\theta_{i}^{\prime}(0)=0$.

In the contact point (the interface among the fictitious two plus two rods), (2) and (4) provide continuity of position and angles

$$
\begin{aligned}
\mathbf{r}_{1}\left(\bar{s}_{1}\right) & =\mathbf{r}_{2}\left(\bar{s}_{2}\right)=\mathbf{r}_{3}\left(\bar{s}_{3}\right)=\mathbf{r}_{4}\left(\bar{s}_{4}\right), \\
\theta_{1}\left(\bar{s}_{1}\right)=\theta_{2}\left(\bar{s}_{2}\right) & =\theta_{3}\left(\bar{s}_{3}\right)+\pi=\theta_{4}\left(\bar{s}_{4}\right)+\pi,
\end{aligned}
$$

and are complemented by balance of forces and torques

$$
\begin{array}{r}
\mathbf{N}_{1}\left(\bar{s}_{1}\right)+\mathbf{N}_{2}\left(\bar{s}_{2}\right)+\mathbf{N}_{3}\left(\bar{s}_{3}\right)+\mathbf{N}_{4}\left(\bar{s}_{4}\right)=0, \\
\frac{d w_{1}}{d \theta_{1}^{\prime}}\left(\bar{s}_{1}\right)+\frac{d w_{2}}{d \theta_{2}^{\prime}}\left(\bar{s}_{2}\right)+\frac{d w_{3}}{d \theta_{3}^{\prime}}\left(\bar{s}_{3}\right)+\frac{d w_{4}}{d \theta_{4}^{\prime}}\left(\bar{s}_{4}\right)=0 .
\end{array}
$$

The extra terms due to the total variations of the fields in the unknown contact point, appearing in (16) in square brackets, can be understood as a continuity of the Legendre transform of the strain 
energy (the Hamiltonian) or, in other contexts, as the continuity of the Eshelby stress $(\mathbf{2}, \mathbf{4}, \mathbf{2 1})$. Using (5) and (20), we eventually find the condition that defines the curvilinear coordinates $\bar{s}_{1}$ and $\bar{s}_{2}$ of the interface point

$$
\begin{aligned}
& {\left[w_{1}-\theta_{1}^{\prime} \frac{d w_{1}}{d \theta_{1}^{\prime}}+\mathbf{N}_{1} \cdot \mathbf{t}_{1}\right]\left(\bar{s}_{1}\right)-\left[w_{3}-\theta_{3}^{\prime} \frac{d w_{3}}{d \theta_{3}^{\prime}}+\mathbf{N}_{3} \cdot \mathbf{t}_{3}\right]\left(\bar{s}_{3}\right)=0,} \\
& {\left[w_{2}-\theta_{2}^{\prime} \frac{d w_{2}}{d \theta_{2}^{\prime}}+\mathbf{N}_{2} \cdot \mathbf{t}_{2}\right]\left(\bar{s}_{2}\right)-\left[w_{4}-\theta_{4}^{\prime} \frac{d w_{4}}{d \theta_{4}^{\prime}}+\mathbf{N}_{4} \cdot \mathbf{t}_{4}\right]\left(\bar{s}_{4}\right)=0,}
\end{aligned}
$$

where relations (7), (15) and (17c) have been used.

The statement of the differential problem is well posed: the system (17) contains four secondorder boundary value scalar equations and eight vectorial first-order equations, requiring altogether 24 boundary conditions provided by (18), (19) and (20). The differential system is fully coupled: despite (17a) and (17b) apparently do not involve the position, their boundary conditions do.

\section{Reduction of the system of equations}

The equation for the unloaded rod $\mathbf{r}_{3}$ and $\mathbf{r}_{4}$ can be readily integrated: (17b) with boundary condition (18a) has solution $\mathbf{N}_{3}\left(s_{3}\right)=\mathbf{N}_{4}\left(s_{4}\right)=0$. Equation (17a) can now be integrated with boundary condition (18b) thus giving $\theta_{3}^{\prime}\left(\bar{s}_{3}\right)=\theta_{4}^{\prime}\left(\bar{s}_{4}\right)=0$; it follows that $\theta_{3}\left(s_{3}\right)$ and $\theta_{4}\left(s_{4}\right)$ are equal to constants to be fixed by the continuity conditions (4). It follows that whatever the actual length of the leaflets $\ell_{1}$ and $\ell_{2}$, the superposition lengths $\ell_{1}-\bar{s}_{1}$ and $\ell_{2}-\bar{s}_{2}$ are immaterial and the condition (5) does not affect the generality of the results. The equations for rods $\mathbf{r}_{3}$ and $\mathbf{r}_{4}$, not subject to pressure, are therefore trivial, and we can concentrate our efforts in solving the equations for $\mathbf{r}_{1}$ and $\mathbf{r}_{2}$. Because of the arguments above, the system of equations and the corresponding boundary conditions can then be rewritten as follows:

$$
\begin{array}{ll}
\frac{d^{2} w_{i}}{d^{2} \theta_{i}^{\prime}} \theta_{i}^{\prime \prime}+\mathbf{N}_{i} \cdot \mathbf{n}_{i}=0, & i=1,2 \\
\mathbf{N}_{1}^{\prime}=p \mathbf{n}_{1}, & \mathbf{N}_{2}^{\prime}=-p \mathbf{n}_{2}, \\
\mathbf{r}_{i}^{\prime}=\mathbf{t}_{i}, & i=1,2 .
\end{array}
$$

The system of equations is supplemented by the boundary conditions

$$
\begin{array}{rr}
\frac{d w_{i}}{d \theta_{i}}(0)=0, & i=1,2 \\
\mathbf{r}_{1}(0)=\mathbf{r}_{1}^{0}, & \mathbf{r}_{2}(0)=\mathbf{r}_{2}^{0} .
\end{array}
$$

In the contact point (the interface among the fictitious four rods) (2) and (4) provide continuity of position and angles

$$
\begin{aligned}
& \mathbf{r}_{1}\left(\bar{s}_{1}\right)=\mathbf{r}_{2}\left(\bar{s}_{2}\right), \\
& \theta_{1}\left(\bar{s}_{1}\right)=\theta_{2}\left(\bar{s}_{2}\right),
\end{aligned}
$$


and are complemented by balance of forces and torques

$$
\begin{aligned}
\mathbf{N}_{1}\left(\bar{s}_{1}\right)+\mathbf{N}_{2}\left(\bar{s}_{2}\right) & =0, \\
\frac{d w_{1}}{d \theta_{1}^{\prime}}\left(\bar{s}_{1}\right)+\frac{d w_{2}}{d \theta_{2}^{\prime}}\left(\bar{s}_{2}\right) & =0 .
\end{aligned}
$$

Finally, the interface conditions (21) simplify to

$$
\begin{aligned}
& w_{1}\left(\theta_{1}^{\prime}\right)\left(\bar{s}_{1}\right)-\frac{d w_{1}}{d \theta_{1}^{\prime}}\left(\bar{s}_{1}\right) \theta_{1}^{\prime}\left(\bar{s}_{1}\right)+\mathbf{N}_{1}\left(\bar{s}_{1}\right) \cdot \mathbf{t}_{1}\left(\bar{s}_{1}\right)=0, \\
& w_{2}\left(\theta_{2}^{\prime}\right)\left(\bar{s}_{2}\right)-\frac{d w_{2}}{d \theta_{2}^{\prime}}\left(\bar{s}_{2}\right) \theta_{2}^{\prime}\left(\bar{s}_{2}\right)+\mathbf{N}_{2}\left(\bar{s}_{2}\right) \cdot \mathbf{t}_{2}\left(\bar{s}_{2}\right)=0 .
\end{aligned}
$$

The complexity of the original system has been significantly reduced: we now have to solve two second-order boundary value problems and four vectorial first-order differential equations, altogether complemented by twelve (coupled) boundary conditions.

\section{Integrals of the motion}

The equations of every rod has an integral of motion. Noting that $\mathbf{n}_{1}=\mathbf{N}_{1}^{\prime} / p$ and $\mathbf{n}_{2}=-\mathbf{N}_{2}^{\prime} / p$, we can write

$$
\begin{aligned}
& \frac{d^{2} w_{1}}{d^{2} \theta_{1}^{\prime}} \theta_{1}^{\prime \prime}+\mathbf{N}_{1} \cdot \frac{\mathbf{N}_{1}^{\prime}}{p}=\left(\frac{d w_{1}}{d \theta_{1}^{\prime}}+\frac{\left|\mathbf{N}_{1}\right|^{2}}{2 p}\right)^{\prime}=0, \\
& \frac{d^{2} w_{2}}{d^{2} \theta_{2}^{\prime}} \theta_{2}^{\prime \prime}-\mathbf{N}_{2} \cdot \frac{\mathbf{N}_{2}^{\prime}}{p}=\left(\frac{d w_{2}}{d \theta_{2}^{\prime}}-\frac{\left|\mathbf{N}_{2}\right|^{2}}{2 p}\right)^{\prime}=0 .
\end{aligned}
$$

A first integral can be used, for instance, to obtain relationships about the value of the unknowns at the endpoints of the rods: because of the arguments illustrated above and exploiting the boundary conditions (18b) and (25b), the sum of the first integrals in (27) evaluated at the endpoints satisfy

$$
\left|\mathbf{N}_{1}(0)\right|^{2}-\left|\mathbf{N}_{2}(0)\right|^{2}=\left|\mathbf{N}_{1}\left(\bar{s}_{1}\right)\right|^{2}-\left|\mathbf{N}_{2}\left(\bar{s}_{2}\right)\right|^{2} .
$$

First integrals play an important role also because if they are functionally independent, that is, their Jacobian matrix has maximal rank, the number of equations of motion can be reduced introducing a new system of coordinates adapted to the foliation generated by the first integrals. To this aim, we first rewrite (22b) and (26) in a slightly different form,

$$
\begin{aligned}
& \left(N_{1}^{x}\right)^{\prime}=-p \sin \theta_{1}, \quad\left(N_{2}^{x}\right)^{\prime}=p \sin \theta_{2}, \\
& \left|\mathbf{N}_{1}\right|^{\prime}=-\frac{p\left(\frac{d w_{1}}{d \theta_{1}^{\prime}}\right) \theta_{1}^{\prime \prime}}{\left|\mathbf{N}_{1}\right|}, \quad\left|\mathbf{N}_{2}\right|^{\prime}=\frac{p\left(\frac{d w_{2}}{d \theta_{2}^{\prime}}\right) \theta_{2}^{\prime \prime}}{\left|\mathbf{N}_{2}\right|},
\end{aligned}
$$


and we give a name to the first integrals:

$$
h_{1}=\frac{d w_{1}}{d \theta_{1}^{\prime}}+\frac{\left|\mathbf{N}_{1}\right|^{2}}{2 p} \quad \text { and } \quad h_{2}=\frac{d w_{2}}{d \theta_{2}^{\prime}}-\frac{\left|\mathbf{N}_{2}\right|^{2}}{2 p} .
$$

Since they are functionally independent, we can compute $\left|\mathbf{N}_{i}\right|$ as a function of $h_{i}$, namely

$$
\left|\mathbf{N}_{1}\right|=\sqrt{2\left(h_{1} p-\frac{d w_{1}}{d \theta_{1}^{\prime}}\right)} \quad\left|\mathbf{N}_{2}\right|=\sqrt{2\left(\frac{d w_{2}}{d \theta_{2}^{\prime}}-h_{2} p\right)} .
$$

Finally, we can rewrite the equation of motion as follows

$$
\begin{aligned}
& \frac{d^{2} w_{1}}{d^{2} \theta_{1}^{\prime}} \theta_{1}^{\prime \prime}-N_{1}^{x} \sin \theta_{1} \pm \cos \theta_{1} \sqrt{2\left(h_{1} p-\frac{d w_{1}}{d \theta_{1}^{\prime}}\right)-\left(N_{1}^{x}\right)^{2}}=0 \\
& \frac{d^{2} w_{2}}{d^{2} \theta_{2}^{\prime}} \theta_{2}^{\prime \prime}-N_{2}^{x} \sin \theta_{2} \pm \cos \theta_{2} \sqrt{2\left(\frac{d w_{2}}{d \theta_{2}^{\prime}}-h_{2} p\right)-\left(N_{2}^{x}\right)^{2}}=0 \\
& \left(N_{1}^{x}\right)^{\prime}=-p \sin \theta_{1}, \quad\left(N_{2}^{x}\right)^{\prime}=p \sin \theta_{2} \\
& h_{1}^{\prime}=0, \quad h_{2}^{\prime}=0 \\
& \mathbf{r}_{i}^{\prime}=\mathbf{t}_{i}, \quad i=1,2 .
\end{aligned}
$$

The system (32) is complemented by the boundary conditions (23-25b) and the ones deriving from the first integral, that is, (28). The plus or minus sign is due to the fact that the level sets defined by the first integrals cannot be parametrized by a global chart, like the sphere, so the sign depends on the initial conditions.

\subsection{The quadratic energy case}

In the particular case of quadratic bending energy

$$
w_{i}\left(\theta_{i}\right)=\frac{k_{i}}{2}\left(\theta_{i}^{\prime}\right)^{2}
$$

where $k_{i}$ is a bending modulus, another first integral exists. After multiplication of equation (17a) by $\theta_{i}^{\prime}$, we can write

$$
\begin{aligned}
k_{i} \theta_{i}^{\prime \prime} \theta_{i}^{\prime}+\mathbf{N}_{i} \cdot \mathbf{n}_{i} \theta_{i}^{\prime} & =\frac{k_{i}}{2} \frac{d}{d s_{i}}\left(\theta_{i}^{\prime}\right)^{2}-\mathbf{N}_{i} \cdot \mathbf{t}_{i}^{\prime} \\
& =\frac{k_{i}}{2} \frac{d}{d s_{i}}\left(\theta_{i}^{\prime}\right)^{2}-\left(\mathbf{N}_{i} \cdot \mathbf{t}_{i}\right)^{\prime}+\mathbf{N}_{i}^{\prime} \cdot \mathbf{t}_{i} \\
& =\left(\frac{k_{i}}{2}\left(\theta_{i}^{\prime}\right)^{2}-\mathbf{N}_{i} \cdot \mathbf{t}_{i}\right)^{\prime}=0
\end{aligned}
$$

A simple calculation shows that this first integral coincides with the interface condition (26). This peculiarity of the quadratic energy (and of the linear bending moment) can be understood observing 
that in (26) it is prescribed the jump of the Legendre transform of the bending energy, but the Legendre transform of a quadratic function is (out of the sign) the function itself. It follows that, for a quadratic energy, the interface conditions prescribe the curvilinear coordinate of the attachment point fixing the value of the first integral. If the value of such a first integral were prescribed as a boundary condition (which is not the case considered herein) two possibilities apply: if the jump condition across $\bar{s}$ is satisfied, that is, true for every $\bar{s}$ and all the interface points are of indifferent equilibrium; otherwise no equilibrium exists.

As we have seen before, first integrals play an important role in reducing the number of equations of motion. We define

$$
\begin{array}{ll}
h_{1}=k_{1} \theta_{1}^{\prime}+\frac{\left|\mathbf{N}_{1}\right|^{2}}{2 p}, & g_{1}=k_{1} \frac{\theta_{1}^{\prime 2}}{2}-\mathbf{N}_{1} \cdot \mathbf{t}_{1}=0, \\
h_{2}=k_{2} \theta_{2}^{\prime}-\frac{\left|\mathbf{N}_{2}\right|^{2}}{2 p}, & g_{2}=k_{2} \frac{\theta_{2}^{\prime 2}}{2}-\mathbf{N}_{2} \cdot \mathbf{t}_{2}=0,
\end{array}
$$

where the fact that $g_{1}$ and $g_{2}$ are zero is exactly the reformulation of the interface (boundary) condition (26), in the case of quadratic energy.

The first integrals $g_{i}$, peculiar of the quadratic energy, provide us with some information a priori about the reaction force at the boundary of the rods. In fact, recalling the boundary condition (18b) and observing that the force balance (20) can be enforced in the contact point only if the tangent reaction forces vanish, we get the additional information:

$$
\mathbf{N}_{i}(0) \cdot \mathbf{t}_{i}(0)=0, \quad \mathbf{N}_{i}\left(\bar{s}_{i}\right) \cdot \mathbf{t}_{i}\left(\bar{s}_{i}\right)=0, \quad \theta_{i}^{\prime}\left(\bar{s}_{i}\right)=0 .
$$

The map $\left(\theta_{i}, \theta_{i}^{\prime}, \mathbf{N}_{i}\right) \mapsto\left(h_{1}, g_{1}, h_{2}, g_{2}\right)$, away from singular points, is a submersion and can therefore be inverted representing $\mathbf{N}_{i}$ as a function of $h_{1}, g_{1}, h_{2}$ and $g_{2}$. Thus a new system of coordinates, adapted to the level sets of the first integrals can be defined in the phase space. By the adhesion condition (26a), (26b) in the case of quadratic energy, we know that the value of the first integrals $g_{1}$ and $g_{2}$ is zero. Thus the system can be rewritten as

$$
\begin{aligned}
& k_{1} \theta_{1}^{\prime \prime} \mp \sqrt{2 p h_{1}-2 p k_{1} \theta_{1}^{\prime}-\frac{k_{1}^{2}}{4} \theta_{1}^{\prime 4}}=0 \\
& k_{2} \theta_{2}^{\prime \prime} \mp \sqrt{-2 p h_{2}+2 p k_{2} \theta_{2}^{\prime}-\frac{k_{2}^{2}}{4} \theta_{2}^{\prime 4}}=0 \\
& h_{i}^{\prime}=0, \quad i=1,2 \\
& \mathbf{r}_{i}^{\prime}=\mathbf{t}_{i}, \quad i=1,2
\end{aligned}
$$

where equations are coupled by the boundary conditions (23a)-(26b). As before, the plus or minus sign is linked to the fact that the level sets of the first integrals cannot be parametrized by a global chart, then the sign depends on the initial conditions.

\section{Asymptotic analysis and critical pressure}

In this section, we explore an approximation of the nonlinear problem obtained by an asymptotic expansion in a small parameter, corresponding to the low pressure. We will show numerically that, 
rather surprisingly, the approximation retains its validity beyond the low pressure regime. This analysis will lead us to determine a critical pressure beyond which there is no more contact of the rods and therefore no more equilibrium solution.

In this section, we restrict to consider the symmetric case and quadratic energy, where the problem can be reduced to the analysis of a single beam, with boundary conditions $\theta^{\prime}(0)=0, \theta^{\prime}(\bar{s})=0$, $\theta(\bar{s})=3 \pi / 2$ and $x(0)=0, y(0)=0$. In this simpler setting, there is no need to consider explicitly the reaction force $\mathbf{N}$, since it can be eliminated using a first integral, and the contact condition (26) is replaced by the condition $x(\bar{s})=d / 2$. To this aim, let us differentiate equation (22a); then using the first integral $g_{2}$ in (35) and the projection of equations (22b) along the normal component, we obtain

$$
k \theta^{\prime \prime \prime}+\frac{k}{2}\left(\theta^{\prime}\right)^{3}-p=0
$$

This decoupled third-order equation is valid out of assumptions, but its applicability is restricted by the knowledge of the needed boundary conditions in $\theta$ and its derivatives; this applies only for the symmetric case assumed herein. For fixed $\bar{s}$, it is known that (38) can be solved in terms of elliptic functions (22-24). However, for our purposes, a more transparent insight of the solution is obtained by a simpler asymptotic analysis.

We can make this equation non-dimensional by introducing the variable $\sigma=s / \bar{s}$, the dimensionless parameters

$$
\alpha=\frac{p d^{3}}{k}, \quad \xi=\frac{\bar{s}}{d},
$$

and the function $\hat{\theta}(\sigma)=\left.\theta(s)\right|_{s=\bar{s} \sigma}$ so that the problem for $\hat{\theta}$ reads

$$
\left\{\begin{array}{l}
\hat{\theta}^{\prime \prime \prime}+\frac{1}{2}\left(\hat{\theta}^{\prime}\right)^{3}-\alpha \xi^{3}=0 \\
\hat{\theta}^{\prime}(0)=0, \quad \hat{\theta}^{\prime}(1)=0, \quad \hat{\theta}(1)=\frac{3}{2} \pi
\end{array}\right.
$$

where the differentiation is taken with respect to the variable $\sigma$. It is worth noticing that we do not know the value of $\xi$ yet, since the contact point $\bar{s}$, as a function of $\alpha$, is unknown. Nonetheless, in order to proceed with the derivation of the asymptotic expansion, it is convenient to assume that $\alpha \xi^{3} \ll 1$ as $\alpha \rightarrow 0$. We will have to check a posteriori that our asymptotic results are compatible with this assumption.

When $\alpha \xi^{3} \ll 1$ it is possible to look for a regular asymptotic expansion (25) of (40). Therefore, we look for a solution of the form $\hat{\theta}(\sigma)=\frac{3}{2} \pi+\sum_{k=1}^{N} \varepsilon^{k} \hat{\theta}_{k}(\sigma)+O\left(\varepsilon^{N+1}\right)$, with $\varepsilon=\alpha \xi^{3}$. By collecting the terms of homogeneous degree in $\varepsilon$, we obtain a chain of linear differential equations that are easily solved. Namely, up to order $O\left(\varepsilon^{3}\right)$ we get

$$
\hat{\theta}_{1}^{\prime \prime \prime}(\sigma)=1, \quad \hat{\theta}_{2}^{\prime \prime \prime}(\sigma)=0, \quad \hat{\theta}_{3}^{\prime \prime \prime}(\sigma)+\frac{1}{2} \hat{\theta}_{1}^{\prime}(\sigma)^{3}=0,
$$

whose solutions, with vanishing boundary conditions, allow us to write $\hat{\theta}(\sigma)$ as

$$
\begin{aligned}
\hat{\theta}(\sigma)= & \frac{3}{2} \pi+\frac{\alpha \xi^{3}}{12}(1-\sigma)^{2}(1+2 \sigma) \\
& +\frac{\left(\alpha \xi^{3}\right)^{3}}{80640}(1-\sigma)^{2}\left(4+8 \sigma+3 \sigma^{2}-2 \sigma^{3}-7 \sigma^{4}-12 \sigma^{5}+25 \sigma^{6}-10 \sigma^{7}\right)+O\left(\left(\alpha \xi^{3}\right)^{5}\right) .
\end{aligned}
$$




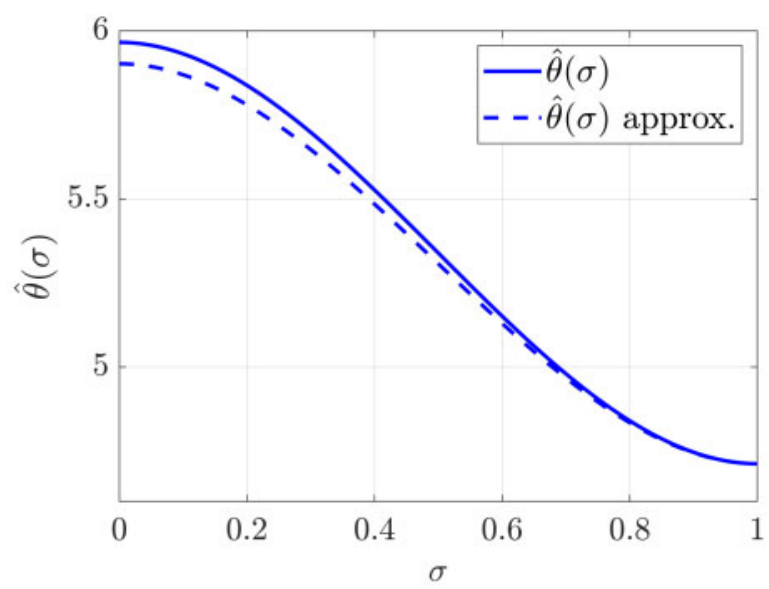

Fig. 2 Comparison between the numerical solution and the approximate angle $\theta$ as given in (42) for $\alpha \approx 15.6$. The numerical solution returns $\bar{s} / d \approx 0.94$ in this case. Hence, we find a relatively large value of $\alpha \xi^{3} \approx 12.8$. Despite this, the asymptotic approximation is still close to the numerical solution.

It can be noticed that boundary conditions, that are zeroth order in $\varepsilon$, are satisfied by the zeroth-order term of the asymptotic expansion, while the higher order contributions and their derivatives vanish at the boundary.

This expression reproduces accurately the numerical solution in $s=\bar{s}$, and works remarkably well also for rather large values of $\alpha \xi^{3}$ (see Fig. 2 for a comparison). However, its error is larger as far as $s$ decreases and its extension to $s \approx 0$ is only valid at low pressures.

We can use (42) to find an explicit approximate evaluation of $\xi=\bar{s} / d$. From the boundary condition $x(\bar{s})=d / 2$ and $x^{\prime}(s)=\cos \theta(s)$ we derive, to leading order,

$$
\frac{d}{2}=\bar{s} \int_{0}^{1} \sin \left(\frac{\alpha \xi^{3}}{12}(1-\sigma)^{2}(1+2 \sigma)\right) d \sigma \sim \bar{s} \int_{0}^{1} \frac{\alpha \xi^{3}}{12}(1-\sigma)^{2}(1+2 \sigma) d \sigma=\bar{s} \frac{\alpha \xi^{3}}{24}
$$

Finally, using $\xi=\bar{s} / d$, we obtain

$$
\xi \sim\left(\frac{12}{\alpha}\right)^{1 / 4}
$$

This result shows that $\alpha \xi^{3} \sim 12^{3 / 4} \alpha^{1 / 4}$, which is compatible with our initial assumption $\alpha \xi^{3} \ll 1$, as $\alpha \rightarrow 0$. Therefore, the asymptotic analysis is consistent.

Equation (42) provides an indication on the asymptotic behaviour of various quantities of interest, such as $\bar{s} / d$ and $\theta(0)$ as far as the rods are in contact. A comparison of (42) with the numerical solution, corresponding to $\alpha \xi^{3} \approx 12.8$ is shown in Fig. 2 . The estimate (44) returns $\bar{s} / d \approx 0.936$ against the numerical result 0.94 .

Remarkably, the approximation (44) is very robust and also applies when the pressure is high. In Fig. 3, we plot the ratio $\bar{s} / d$ against the dimensionless pressure difference. We observe that the simple asymptotic approximation (44) captures the correct behaviour also for relatively large pressures. It 


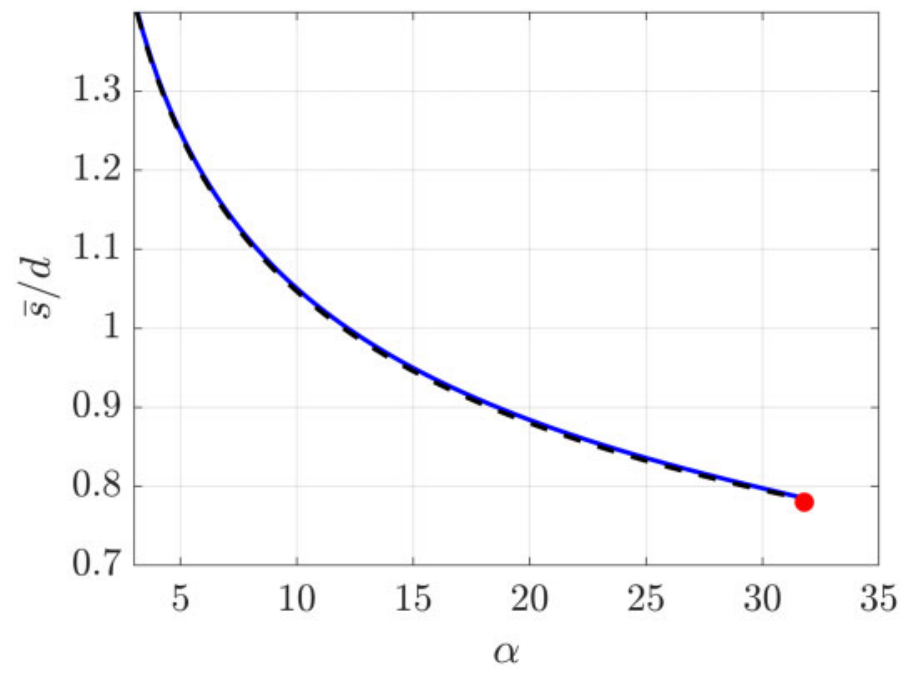

Fig. 3 Arc length of the contact point as a function of $\alpha$. For comparison, the dashed line shows the asymptotic approximation $\xi \sim(12 / \alpha)^{1 / 4}$, as given in (44). Red dot shows the limit point $\left(\alpha_{c r}, \xi_{c r}\right) \approx(31.8,0.78)$.

is therefore tempting to look for an approximation of (40) which is uniformly valid in [0, 1]. To this aim, we consider the family of functions of the form

$$
\hat{\theta}(\sigma)=\frac{3}{2} \pi+A(1+\cos (\pi \sigma)),
$$

parametrized by the amplitude $A$. Furthermore, we observe that (40) is the Euler-Lagrange equation of the reduced functional

$$
W_{\mathrm{r}}\left(\hat{\theta}^{\prime}\right)=\int_{0}^{1}\left(\frac{1}{2}\left(\hat{\theta}^{\prime \prime}(\sigma)\right)^{2}-\frac{1}{8}\left(\hat{\theta}^{\prime}(\sigma)\right)^{4}+\alpha \xi^{3} \hat{\theta}^{\prime}(\sigma)\right) d \sigma,
$$

so that, in our family of functions, we look for the amplitude $A$ that makes $W_{\mathrm{r}}$ stationary. This way, we identify the value of $A$ that approximates at best a solution within the considered functional space. The substitution of (45) into (46) yields, after an explicit integration, a function of the amplitude $A$

$$
g(A)=-\frac{3 \pi^{4}}{64} A^{4}+\frac{\pi^{4}}{4} A^{2}-2 \alpha \xi^{3} A,
$$

whose critical points identify the values of $A$. The analysis of the discriminant of $g^{\prime}(A)=0$ reveals that there are three real critical points if $\alpha \xi^{3} \leq \sqrt{2} \pi^{4} / 9$. By contrast, when $\alpha \xi^{3}>\sqrt{2} \pi^{4} / 9$ there is only one (negative) real solution, which, however, must be rejected since it cannot represent our physical situation. This implies, after substitution of (44), that there is a critical dimensionless pressure, $\alpha_{c r}$, above which the contact solution disappears

$$
\alpha \leq \alpha_{c r}=\left(\frac{\pi^{8}}{972 \sqrt{3}}\right)^{2} \approx 31.8
$$



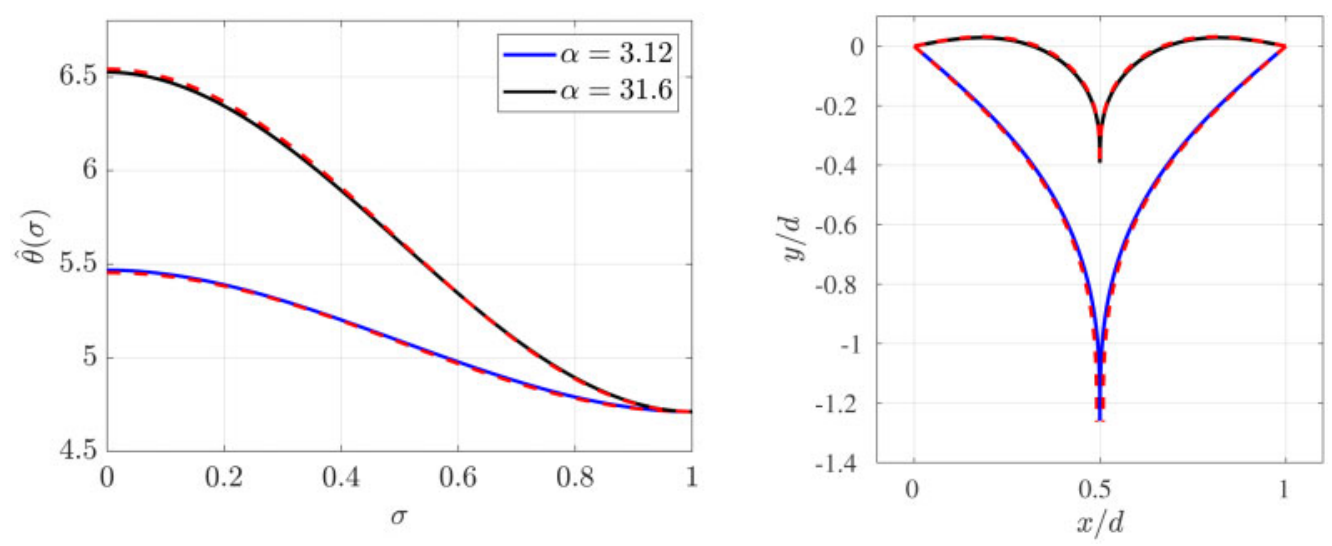

Fig. 4 Comparison between the numerical solutions (solid lines) and the approximation (45) (dashed red lines), with the amplitude as given in (49), for $\alpha \approx 3.12$ (blue) and $\alpha \approx 31.6$ (black). On the right figure, only the free parts of the rods are shown.

a value that is in excellent agreement with the simulations. Correspondingly, (44) yields a critical value $\xi: \xi_{c r} \approx 0.78$. When $\alpha \leq \alpha_{c r}$, the amplitude that approximates at best our numerical solution is one of the critical values of $g(A)$ and it is explicitly found to be

$$
A=\frac{2}{3} \sqrt{2}\left(\cos \left(\frac{1}{3} \arctan \beta\right)-\sqrt{3} \sin \left(\frac{1}{3} \arctan \beta\right)\right),
$$

with

$$
\beta=\sqrt{\frac{\pi^{8}}{972 \sqrt{3 \alpha}}-1 .}
$$

A comparison of the numerical solution and (45), for two different values of $\alpha$, is plotted in Fig. 4.

\section{Numerical simulations}

In this section, we report the results of the numerical integration of the equations for the quadratic energy discussed in Section 3. Numerical simulations are carried out for possibly different values of the bending stiffness of the two rods, thus corresponding to equal or different values of the dimensionless parameter $\alpha$, denoted in the sequel as $\alpha_{i}=p d^{3} / k_{i}(i=1,2)$. The values of $\alpha_{i}$ adopted in the simulations are representative of the low pressure regime and near to the critical pressure regime, as found in the previous section. Numerical integration is performed using the package $b v p 4 c$ of Matlab which solves a boundary value problem by multistep finite differences controlling the discrete relative error to be below the tolerance of $10^{-8}$. Another, indirect, check of the consistency of the numerical results is obtained comparing the computed curvilinear coordinate $\bar{s}$ of the detachment point with the one obtained by direct integration of the arc length $\sqrt{x^{\prime 2}+y^{\prime 2}}$.

Figure 5 plotted the shape of the rods at equilibrium for a symmetric system $\left(\alpha_{1}=\alpha_{2}\right)$ for different values of the dimensionless parameter. The increasing pressure raises the $y$ coordinate of the contact points and increases the curvature of the rods. 

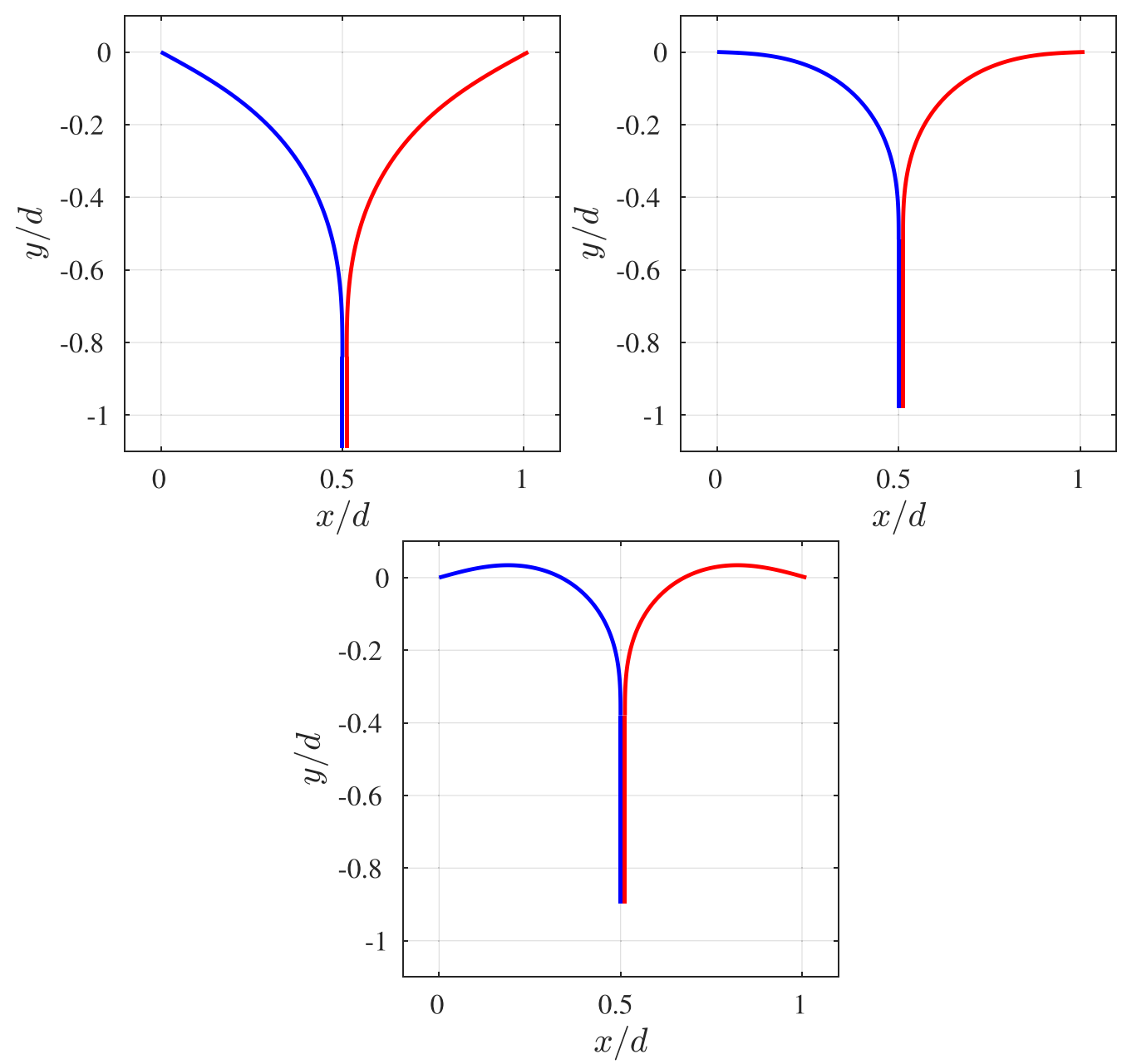

Fig. 5 Symmetric system of rods in contact: the two leaflets have the same stiffness. Plots correspond to $\alpha=10 ; 25 ; 32$. The increasing pressure raises the $y$ coordinate of the contact point and generates a greater curvature of the rods.

Figure 6 shown the reaction forces $\mathbf{N}_{1}(s)$ and $\mathbf{N}_{2}(s)$ computed for $\alpha_{1}=\alpha_{2}=32$ (symmetric case) and $\alpha_{1}=26.6, \alpha_{2}=44.2$ (non-symmetric system). As expected after the remark (36), the tangent component of the reaction forces vanishes at the boundaries.

A plot of the profile with non-symmetric rods subject to pressure is shown in Fig. 7 for $\left(\alpha_{1}=\right.$ 37, $\left.\alpha_{2}=22\right)$, and $\left(\alpha_{1}=44, \alpha_{2}=26\right)$ : the difference in stiffness generates a non-vertical orientation of the overlapping region. Such an angle does not depend on the length of the rods. We note that in the non-symmetric case, we are able to find a solution for values of $\alpha_{1}$ greater than the critical pressure corresponding to the symmetric case. This might lead us to conjecture that an non-symmetric 

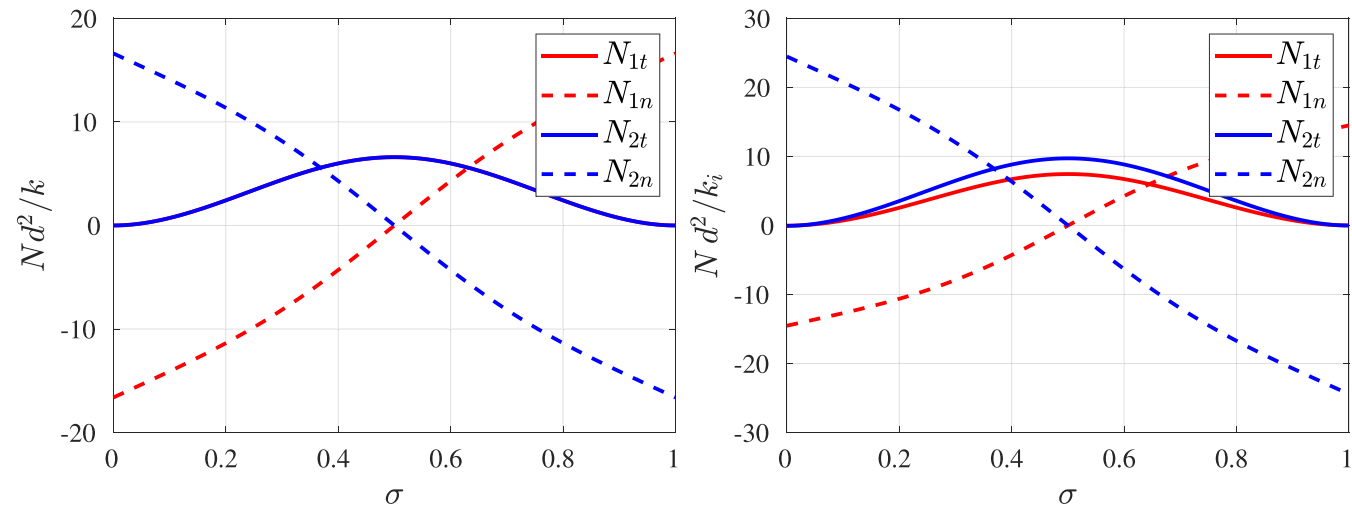

Fig. 6 Plot of the reaction force $\mathbf{N}_{1}$ and $\mathbf{N}_{2}$, measured in units of $k_{i} / d^{2}$, computed with $\alpha_{1}=\alpha_{2}=32$ (left) and $\alpha_{1}=44, \alpha_{2}=26$ (right). The tangent $\left(N_{t}\right)$ and normal $\left(N_{n}\right)$ components are plotted versus the curvilinear coordinate $s$ for a symmetric (left) and non-symmetric (right) rod system.
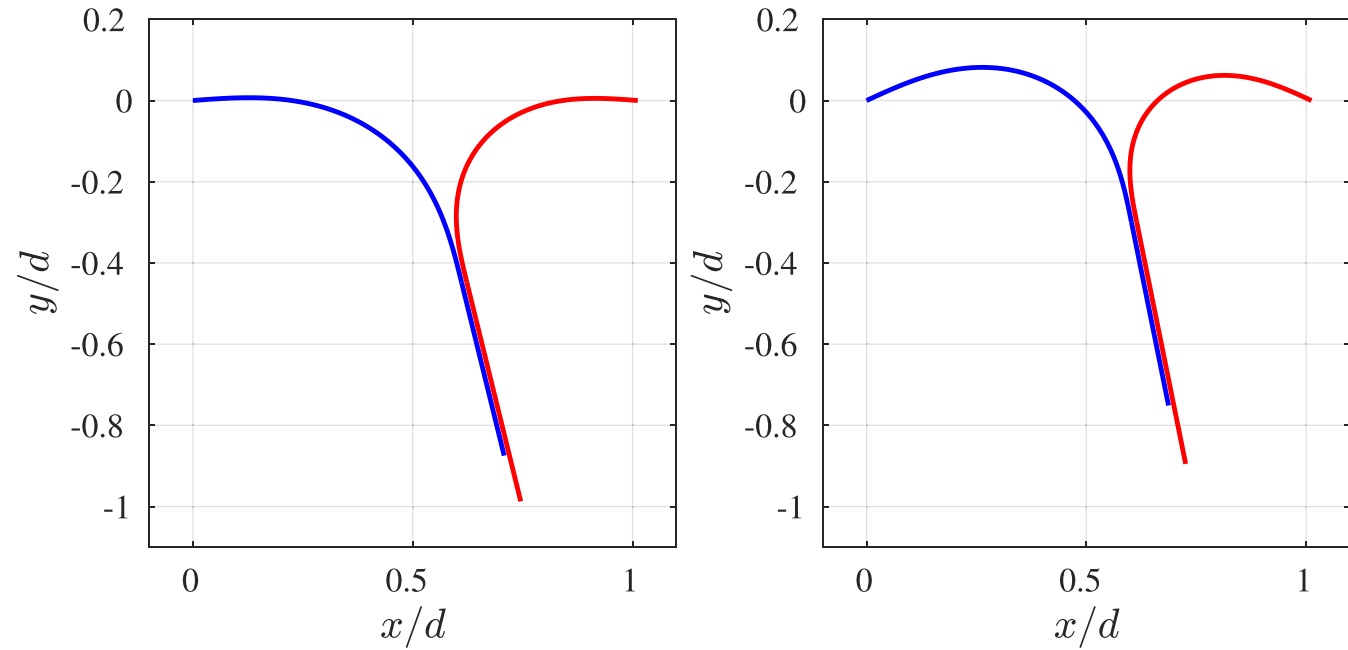

Fig. 7 Non-symmetric beams: the difference in stiffness generates a non-vertical orientation of the overlapping region. Numerical values of the parameter: $\left(\alpha_{1}, \alpha_{2}\right)=(37,22)$ and $\left(\alpha_{1}, \alpha_{2}\right)=(44,26)$.

configuration can sustain higher pressures, a remark that could have consequences in terms of optimal design that remain to be investigated.

\section{Conclusions}

We have studied the mechanical problem of two inextensible rods with a fixed point, partially in contact, subject to a pressure difference. After variational statement of the problem, we have 
obtained the balance equations of the system with the corresponding boundary conditions, and the jump conditions that define the curvilinear coordinate of the contact point. System reduction has been carried out exploiting first integrals, in particular in the case of quadratic bending energy. Asymptotic analysis yields approximate solutions that fit very well with the fully nonlinear theory. In particular, we have been able to determine in a closed (approximate) way the value of the pressure that discriminates the closed regime (when the two rods are in contact) from the open one (when the pressure is too large to be sustained). The numerical simulations show the configuration of the rods for values of the pressure below the critical one.

The problem discussed in this article has a theoretical relevance as a simplest paradigm apt to study the contact of elastic bodies in the presence of large deformations. While the interaction of a single elastic ring confined by rigid external container has been extensively studied (5-7), few results are available for elastic rods confined by their mutual interaction (8-10). The present contribution can be the basis for extension of the model to more complex physical systems where interaction potentials or more complex constraints intervene.

\section{Acknowledgements}

The authors acknowledge the support from the Italian Ministry of University and Research through the Grant No. 2017KL4EF3 Mathematics of active materials: from mechanobiology to smart devices. DA and MZ acknowledge the partial support of the MIUR grant Dipartimenti di Eccellenza 20182022 (E11G18000350001).

\section{References}

1. S.S. Antman. Nonlinear Problems of Elasticity. (Springer, New York NY 2005).

2. C. Majidi, Remarks on formulating an adhesion problem using Euler's elastica (draft), Mech. Res. Commun. 34 (2007) 85-90.

3. C. Majidi, O. M. O'Reilly and J. A. Williams, On the stability of a rod adhering to a rigid surface: shear-induced stable adhesion and the instability of peeling, J. Mech. Phys. Solids 60 (2012) 827-843.

4. O. M. O'Reilly, Modeling Nonlinear Problems in the Mechanics of Strings and Rods. (Springer International, Cham, Switzerland 2017).

5. R. De Pascalis, G. Napoli and S. Turzi, Growth-induced blisters in a circular tube, Physica D 283 (2014) 1-9.

6. G. Napoli and S. S. Turzi, Snap buckling of a confined thin elastic sheet, Proc. R. Soc. A 471 (2015) 20150444.

7. A. L. Hazel and T. Mullin, On the buckling of elastic rings by external confinement, Philos. Trans. R. Soc. A 375 (2017) 20160227.

8. G. Napoli and A. Goriely, A tale of two nested elastic rings, Proc. R. Soc. A 473 (2017) 20170340.

9. F. Lombardo, A. Goriely and G. Napoli, Asymmetric equilibria of two nested elastic rings, Mech. Res. Commun. 94 (2018) 91-94.

10. J. E. Flaherty and J. B. Keller, Contact problems involving a buckled elastica, SIAM J. Appl. Math. 24 (1973) 215-225.

11. M. Moakher and J. H. Maddocks, A double-strand elastic rod theory. Archive Rational Mech. Anal. 177 (2005) 53-91. 
12. E. L. Starostin and G. H. M. van der Heijden, Theory of equilibria of elastic 2-braids with interstrand interaction, J. Mech. Phys. Solids 64 (2014) 83 - 132.

13. D. Vella, J. Bico, A. Boudaoud, B. Roman and P. M. Reis, The macroscopic delamination of thin films from elastic substrates, Proc. Natl. Acad. Sci. USA 106 (2009) 10901-10906.

14. L. Wang, S. M. Roper, N. A. Hill and X. Luo, Propagation of dissection in a residually-stressed artery model, Biomechan. Model. Mechanobiol. 16 (2017) 139-149.

15. L. Wang, N. A. Hill, S. M. Roper and X. Luo, Modelling peeling-and pressure-driven propagation of arterial dissection, J. Eng. Math. 109 (2018) 227-238.

16. P. Litewka and P. Wriggers, Frictional contact between $3 \mathrm{~d}$ beams, Comput. Mech. 28 (2002) 26-39.

17. D. Durville, Contact-friction modeling within elastic beam assemblies: an application to knot tightening, Comput. Mech. 49 (2012) 687-707.

18. D. M. Espino, D. E. T. Shepherd and D. W. L. Hukins, Evaluation of a transient, simultaneous, arbitrary Lagrange-Euler based multi-physics method for simulating the mitral heart valve, Comput. Methods Biomech. Biomed. Eng. 17 (2014) 450-458.

19. I. M. Gelfand and S. V. Fomin, Calculus of Variations. (Prentice-hall 1963 Englewood Cliffs, New Jersey; Dover reprint 2012).

20. A. Goriely, The Mathematics and Mechanics of Biological Growth, Vol. 45. (Springer, New York 2017).

21. G. Napoli and S. S. Turzi, The delamination of a growing elastic sheet with adhesion, Meccanica, 52 (2017) 3481-3487.

22. I. Tadjbakhsh and F. Odeh, Equilibrium states of elastic rings, J. Math. Anal. Appl., 18 (1967) 59-74.

23. V. M. Vassilev, P. A. Djondjorov and I. M. Mladenov, Cylindrical equilibrium shapes of fluid membranes, J. Phys. A 41 (2008) 435201.

24. L. Giomi, Softly constrained films, Soft Matter 9 (2013) 8121-8139.

25. C. M. Bender and S. A. Orszag. Advanced Mathematical Methods for Scientists and Engineers I: Asymptotic Methods and Perturbation Theory. (Springer-Verlag New York 2013). 